Teknokultura. Revista de Cultura Digital y Movimientos Sociales

ISSNe: $1549-2230$

http://dx.doi.org/10.5209/TEKN.69477

\title{
¿Otro dinero es posible? Conexiones entre la mercancía dineraria dentro de la teoría del valor-trabajo de Das Kapital, el bitcoin y la descentralización de la economía
}

\author{
Cristopher Morales Bonilla ${ }^{1}$
}

Recibido: 14 de mayo de 2020 / Aceptado: 30 de junio de 2020 Open peer reviews

\begin{abstract}
Resumen. En las últimas décadas, el nacimiento de las criptomonedas ha venido a desafiar el monopolio del papel moneda controlado por los bancos centrales nacionales y sus respectivos Estados. A partir de una concepción descentralizadora de la economía, monedas digitales como el Bitcoin han intentado sustituir al dinero tradicional como una nueva forma de relación económica más democrática. Sin embargo, es necesario confrontar estos nuevos modelos de intercambio económico con los análisis de Karl Marx en Das Kapital para comprobar si, realmente, suponen una alternativa eficaz al capitalismo o si recaen en formas nuevas de relaciones capitalistas.

Palabras clave: criptomoneda; dinero; economía descentralizada; horizontalidad; Marx.

\section{[en] Value, money and crypto-currency. Connections between money within Das Kapital's labor-value theory, bitcoin and the decentralization of the economy}

\begin{abstract}
In recent decades, the birth of crypto-currency has challenged the monopoly of paper money controlled by national central banks and their respective states. From a decentralized conception of the economy, digital currencies such as Bitcoin have tried to replace traditional money as a new and more democratic form of economic relationship. However, it is necessary to confront these new forms of economic exchange with Karl Marx's analyses in Das Kapital to see whether they really represent an effective alternative to capitalism or whether they fall into new forms of capitalist relations.
\end{abstract}

Keywords: crypto-currency; decentralized economy; horizontality; Marx; money.

Sumario. 1. Introducción. 2. La mercancía dineraria en Das Kapital. 3. ¿El bitcoin como moneda "anticapitalista”? 4. Conclusiones. 5. Referencias.

Cómo citar: Morales Bonilla, C. (2020). ¿Otro dinero es posible? Conexiones entre la mercancía dineraria dentro de la teoría del valor-trabajo de Das Kapital, el bitcoin y la descentralización de la economía. Teknokultura. Revista de Cultura Digital y Movimientos Sociales, 17(2), 141-148.

\section{Introducción}

'Capitalismo digital' es un concepto que parece situarse, a la vez, entre dos momentos históricos diferentes. Por un lado, remite al modo de producción que empezó a desarrollarse a comienzos del siglo XIX en Europa, y a partir del cual la humanidad entró en una fase, desconocida hasta entonces, de producción de riquezas. Por otro lado, remite a la digitalización, proceso social, tecnológico, económico y ecológico a través del cual la esfera creada por la computación e Internet ha hecho entrar a la humanidad, otra vez, en una nueva fase de su historia. Una de las preguntas clave que arroja esta nueva etapa del capitalismo tiene que ver con su relación con el pasado: ¿La era de la computación y la cibernética cons- tituye simplemente un nuevo momento dentro de una línea continua, o supone un salto cualitativo que conforma una nueva época histórica, la cual haríamos bien en dejar de llamar 'capitalismo'?

La primera opción es aquella por la que ha optado esa tradición de análisis social, y de estrategia y táctica de transformación política, que ve en el capitalismo, especialmente en el análisis que hace Karl Marx de la crítica de la economía política, el punto de no retorno de la humanidad. Según este punto de vista, el capitalismo es aquel conjunto de elementos, determinados y relacionados al modo en el que Marx los describe a lo largo de su obra, y del cual solo se podrá salir cuando se supere dicho modo de producción a través de la creación de una sociedad socialista o comunista. 
La segunda opción entiende que, tal vez, sea necesario entender la importancia de ese elemento cualitativo que supone el salto digital. Con él, habríamos entrado en una época en la que ciertos conceptos, esquemas, relaciones y determinaciones sociales se habrían transformado para dar paso a un modo de producción de riqueza que, aun manteniendo la explotación, la desigualdad y la amenaza ecológica completamente vivas, se habría reconstituido de tal forma que habría dado paso a otra configuración social que, posiblemente, tendríamos que dejar de llamar capitalismo.

Sin duda, elegir entre una y otra determina absolutamente la forma de entender la naturaleza del capitalismo digital, a la vez que todo lo que está relacionado con él: nuevas formas de explotación, desaparición de la fábrica como unidad central de la producción económica, cuestionamiento de conceptos clave del marxismo, etc. Pero es especialmente importante para poder dar respuesta a la pregunta principal: ¿qué es una sociedad emancipada? A partir de aquí, es posible entender las diversas ramificaciones del capitalismo digital. Sin embargo, hay un campo de problemas, un tipo de relación social, abierto por esta nueva forma de producción, en el que se puede empezar a dar respuesta a la pregunta por la emancipación. Ese campo es el de las criptomonedas y su relación con el dinero tradicional.

Las criptomonedas, especialmente el Bitcoin, son uno de los mejores ejemplos de la ambigüedad y de lo problemático que es el capitalismo digital en relación a los proyectos de emancipación. Lanzan un desafío para el proyecto de la transformación social: ¿supone algún tipo de ganancia una moneda que esté absolutamente fuera de los circuitos de la economía capitalista institucional? ¿Qué relación tiene la posibilidad de una moneda semejante con una sociedad emancipada? Para poder entender la relación entre las criptomonedas y la formulación de un proyecto de transformación social a partir del nuevo contexto del capitalismo digital es necesario entender, en primer lugar, en qué consiste el dinero y cómo funciona dentro del modo de producción capitalista. Para eso, es necesario entender los análisis de Marx sobre el dinero en Das Kapital.

\section{La mercancía dineraria en Das Kapital}

A pesar de que la cuestión del dinero atraviesa toda la obra de Marx, es en Das Kapital donde parece desplegarse de una forma más o menos definitiva. A la vez, intenta resolver otro problema que, en el fondo, parece mucho más importante y que es el que le lleva a interesarse por el valor: ¿cómo es posible establecer una relación de equivalencia, que es aquella que demanda la existencia de un mercado, ya sea capitalista o no, entre dos o más productos, cuya naturaleza es absolutamente diferente entre sí?

Es posible intentar analizar el problema del dinero desde muchas perspectivas. Sin embargo, todas ellas incluyen una forma muy determinada de entender el papel del dinero dentro del proyecto de emancipación que está intentando construir Marx en Das Kapital, cuestión esta que, a veces, ha parecido pasarse por alto, reduciendo a Marx a un científico social cuya única intención sería la de demostrar los hechos en su pura objetividad (De Brunhoff, 1973; Kennedy, 2006; Lange, 2019; Moseley, 2005, 2016; Nelson, 1999; Saad-Filho, 2019). Sin embargo, es posible establecer una forma de abordar el problema del dinero a partir de una pregunta muy concreta: ¿qué naturaleza tiene el dinero y cómo determina, si es que lo hace, el proyecto de emancipación de Marx?

\subsection{La forma simple 0 accidental del valor}

La primera forma es la forma simple o singular de valor. Marx entiende que este es el suelo de la cuestión de la intercambiabilidad. Así, la relación más simple de valor que se puede dar es la que existe entre una mercancía y otra, siendo ambas cualitativa y cuantitativamente diferentes entre sí. Sin embargo, ya desde el comienzo Marx quiere dejar claro que no se trata meramente de una ecuación, de la expresión igual de dos productos del trabajo absolutamente diferentes, sino de la expresión de una mercancía en el valor de otra:

El lienzo expresa su valor en la chaqueta; la chaqueta hace las veces de material para dicha expresión del valor. A la primera mercancía le corresponde un papel activo; a la segunda, uno pasivo. El valor de la primera mercancía queda representado como valor relativo, o sea, reviste una forma relativa de valor. La segunda funciona como equivalente, esto es, adopta una forma de equivalente (Marx, 1991, p. 50$)^{2}$

Marx entiende que, en esta primera forma de la intercambiabilidad, no se trata de igualar dos cosas entre sí de un modo simplista. Tal operación no es posible por la sencilla razón de que no existe un criterio claro del valor de ninguna de las dos mercancías cualitativa y cuantitativamente diferentes. Por ese motivo, una sirve de equivalente de la otra; de medida con respecto a la otra. En este caso, es el lienzo la mercancía que usa Marx como valor básico a partir del cual poder establecer las relaciones de igualdad: el valor de una mercancía corresponde a la cantidad de trabajo socialmente necesario para su producción, la cual, a su vez, está determinada también por el desarrollo 'histórico' de las fuerzas productivas.

Pero lo importante aquí en relación con el dinero es otro elemento. Al no establecer una igualdad equilibrada entre dos mercancías absolutamente diferentes entre sí, sino al situar un esquema en el que una mercancía sirve de referencia de la otra, al establecer una relación de pasividad-actividad entre las mercancías, comienza a desplegarse la lógica del dinero: la diferencia entre mercancías que son diferentes entre sí se resuelve con la aparición de una mercancía que sirva de referencia, de base, de expresión del valor común de esas diferencias.

Todas las citas de fuentes originales han sido traducidas por el autor 


\subsection{La forma desarrollada del valor}

La segunda forma es la 'forma total o desplegada de valor'. En este segundo estadio, las mercancías empiezan a relacionarse entre sí de una forma múltiple. Ya no se trata de la limitación de dos mercancías cualitativa y cuantitativamente diferentes entre sí. Si el estadio anterior parecía poder limitarse a un esquema de trueque, en este caso empieza a vislumbrarse la aparición del mercado moderno. Al multiplicarse las cualidades y las magnitudes, el trueque se hace cada vez más complejo.

No obstante, el principal problema que enuncia Marx en esta fase es el de la infinita serie de expresiones de valor de las mercancías en un esquema semejante. Cualquier cantidad determinada de una mercancía podrá ser intercambiada por infinitas cantidades de infinitos tipos diferentes de mercancías. De este modo, la mercancía que ha tomado Marx como referencia, en este caso el lienzo, seguiría operando del mismo modo, es decir, que seguiría teniendo un papel activo, mientras que el resto de las mercancías tendrían el papel pasivo de constituir la expresión del valor del lienzo, no el propio valor (Marx, 1991, pp. 63-65).

A pesar de que Marx dedica pocas páginas a esta segunda forma de valor (Marx, 1991, pp. 63-65) lo cierto es que tiene una importancia mucho mayor para entender la naturaleza del dinero de lo que podría parecer a simple vista. Básicamente, la principal consecuencia de este esquema es que demuestra la imposibilidad del trueque a nivel social, ya que cualquier intercambio simple de mercancías como el de la primera forma de valor se enfrenta siempre al problema de que esa misma cantidad de mercancías será, a su vez, puesta eternamente en relación con otro conjunto de mercancías cualitativa y cuantitativamente diferentes por parte de cada uno de los participantes en el trueque.

\subsection{La forma general del valor}

La tercera forma de expresión del valor es lo que Marx llama "el carácter modificado de la forma de valor". Las mercancías representan aquí su valor de dos maneras: por un lado, de 'manera simple', es decir, en una sola mercancía, y de 'manera unitaria', es decir, general. En este esquema, un conjunto de mercancías diferentes entre sí se reduce a la mercancía que Marx toma como referente. Justamente, aquí desaparece el problema del salto de las equivalencias, es decir, el problema de que la cadena de equivalencias acaba por romperse justo en el momento en que se deja de tomar como referencia el valor de la mercancía que sirve como base de cálculo.

De alguna forma, aunque Marx no lo explicita (Marx, 1991, pp. 63-64), se trata del momento en el que se intenta resolver el problema de la infinitud de la cadena de equivalencias. No existe otra forma de hacerlo que poner a una mercancía determinada como base universal de referencia. Es necesario que toda mercancía pueda expresarse en una cantidad determinada de la mercancía universal, en este caso el lienzo. Sin duda, se trata del momento en que se despliega definitivamente cómo funcionará el dinero: como un tipo de referente al que todas las mercancías disponibles en un mercado pueden reducirse, con la finalidad de poder determinar en todo momento el valor de cada una de ellas con relación a su cantidad y a su cualidad.

La confirmación de la importancia de este momento viene dada por un pasaje en el que Marx parece situar históricamente este momento de despliegue del esquema: "Es obvio que esta forma, en la práctica, solo se da en los más tempranos comienzos, cuando los productos del trabajo se convierten en mercancías a través de un intercambio fortuito y ocasional" (Marx, 1991, p. 63). La aparición de la necesidad de una mercancía universal parece darse en aquella época pasada en la que el mercado era una forma de relación social más bien fortuita y ocasional. De este modo, Marx sitúa ya el análisis en la necesidad de la aparición del dinero.

\subsection{La forma dinero del valor}

La cuarta forma de valor es el 'dinero'. En esta nueva forma de valor, o relación de valor, se produce un salto cualitativo: se aísla una mercancía concreta, el oro, la cual toma la forma de un equivalente general. Sin duda, y en coherencia con el desarrollo anterior, antes de poder llegar a ser una forma de equivalente general, el oro tuvo que ser, al comienzo de su entrada en el mercado, una mercancía igual que las demás. Pero el hecho de que se eligiera el oro no fue de modo casual: al ser una de las mercancías más difíciles de conseguir, al no estar disponible de manera inmediata en el mercado, el oro acabó por convertirse en la mercancía dineraria por excelencia, es decir, en el dinero (Di Muzio y Robbins, 2017; Marx, 1991, p. 70).

Pero este es solo el primer paso del proceso desde el problema de la intercambiabilidad y del valor hasta la aparición definitiva del dinero tal y como lo manejamos en nuestra vida cotidiana. Marx sitúa justo después de la institución del oro como mercancía dineraria a los precios como expresión del valor de una mercancía a través de dicha mercancía dineraria. La aparición, y hegemonía, del oro como mercancía dineraria universal permite la expresión del valor en el precio: "La expresión relativa simple del valor de una mercancía, por ejemplo, del lienzo, en la mercancía que ya funciona como mercancía dineraria, por ejemplo, en el oro, es la forma de precio" (Marx, 1991, p. 70).

En el texto de Das Kapital, esta primera sección en la que Marx intenta establecer la conexión entre la teoría del valor trabajo y su ulterior desarrollo en el dinero y en los precios termina aquí con una serie de interrogantes que intentará tratar más adelante en el texto, especialmente en la relación entre el dinero, su circulación y su conversión en capital (Marx, 1991, p. 82 y ss.). Sin embargo, este desarrollo posterior se encuadra, precisamente, en el momento histórico determinado en el que Marx intenta explicar cómo funciona el dinero dentro del capital, dentro de sus determinaciones en una forma social concreta.

Pero la relación que se puede establecer con la aparición de las criptomonedas no puede encontrar en el análisis históricamente determinado del dinero, dentro 
de la circulación capitalista, un momento privilegiado a través del cual se pueda entender su naturaleza. El problema aquí radica, principalmente, en que las criptomonedas son un intento de establecer un modo de intercambio monetario fuera o más allá de la forma capitalista. Obviamente, como se verá a continuación, ese carácter anticapitalista de las criptomonedas hay que entenderlo en un sentido muy determinado: sin duda, no bajo la perspectiva de una sociedad ya enteramente capitalista, sino en el sentido estricto de una moneda que se sitúa fuera del circuito de control de aquellas instituciones que producen, controlan y gestionan la distribución del dinero.

Por lo tanto, establecer la relación entre la teoría del valor trabajo y la naturaleza de las criptomonedas tiene por objetivo esclarecer un problema fundamental: cómo es posible, si es que lo es, crear una forma de mercancía dineraria fuera de los circuitos del capital pero que, a la vez, pueda conectarse con el proyecto de emancipación que Marx intenta desarrollar en Das Kapital. Dicho de otro modo: si, tal y como Marx afirma, el momento de circulación del dinero, y su conversión en capital, son procesos que solo se pueden entender 'después' de haber establecido la relación entre la teoría del valor-trabajo y la aparición del dinero, es dicha teoría la que tiene que servir como el punto de comparación con el intento de crear una forma de moneda fuera de los circuitos del capital.

El principal elemento que se puede extraer del desarrollo que hace Marx de las cuatro formas del valor es el carácter histórico que establece para dicho proceso. A pesar de que, por momentos, pueda parecer que Marx está analizando momentos abstractos de un desarrollo conceptual de cómo funciona el dinero, en realidad en todo momento está intentando mostrar ciertos momentos determinados del desarrollo histórico del dinero. Así, la primera forma del valor puede identificarse con el trueque entre dos propietarios de objetos, ni siquiera poseedores de mercancías; la segunda forma se da entre el mismo número de poseedores, todavía fuera del mercado, cuando quieren intercambiar cantidades diversas de mercancías diferentes; la tercera forma aparece en las primeras apariciones históricas de la mercancía, cuando la cadena de equivalencias no parece solucionar el problema del valor de las mercancías; $y$, en último lugar, la mercancía dineraria aparece justo en el momento en que el mercado, todavía no plenamente capitalista, necesita de una mercancía autónoma al resto a partir de la cual se puedan establecer los precios de los productos.

Por lo tanto, es posible establecer una conexión entre una configuración social y material concreta con un desarrollo igual de concreto del problema de la equivalencia de valores, de su intercambiabilidad $\mathrm{y}$, en último término, del problema del dinero. Si este elemento concreto e histórico se debe mantener en el análisis de las formas sociales, ¿no sería posible, en principio, establecer la posibilidad de una forma diferente de dinero, la cual esté en relación directa con las configuraciones contemporáneas del modo de producción capitalista $\mathrm{y}$ que, incluso, pueda desafiar, de alguna forma, la hege- monía de la mercancía dineraria? Esta es precisamente la intención con la que nació el Bitcoin.

\section{3. ¿El bitcoin como moneda "anticapitalista"?}

\subsection{Funcionamiento y nacimiento del bitcoin y del blockchain}

A principios de 2009, justo al comienzo de una de las mayores crisis financieras de los últimos siglos, Satoshi Nakamoto (pseudónimo) publica un artículo en Internet titulado Bitcoin: A Peer-to-Peer Electronic Cash System (Nakamoto, 2009). En él, se explica el modelo de funcionamiento de un tipo de dinero virtual, una criptomoneda, que tendría dos características básicas: por un lado, estaría fuera del circuito de control de las instituciones que producen, gestionan y controlan la producción y circulación del dinero 'real' (bancos centrales y privados, y Estados), y, por otro lado, se basaría en un sistema controlado por algoritmos, el cual supone una forma horizontal y descentralizada de monitorización de las transacciones (Ammous, 2018; Bjerg, 2016; Davidson; De Filippi; Potts, 2018; Franco, 2015; Villareal Robledo, 2016).

Por lo tanto, Bitcoin nace como una forma de resolver el problema de la 'confianza'. A pesar de que el uso y creación del Bitcoin es complejo, lo cual será, como se verá más adelante, uno de sus principales defectos, es necesario entender de forma básica cómo funciona la creación de esta nueva criptomoneda. El sistema descrito por Nakamoto es el de una red peer-to-peer (P2P) cuyo propósito principal es el de promover la creación de transacciones a nivel virtual que requieran de la validación de todos los participantes en la red de creación. De este modo, el componente fundamental del Bitcoin es el de un intercambio entre varios inputs que se aplican a uno o más outputs, y que se comporta igual que las entradas en un libro de contabilidad de doble entrada (Huckle, 2016, pp.2-6).

En esencia, las transacciones de Bitcoins consisten en que personas (o nodos) que poseen Bitcoins informan a la red del cambio de propiedad de sus monedas virtuales al resto de sus miembros. Estos nuevos poseedores pueden, por su parte, crear otra transacción que, a su vez, autoriza futuras transferencias. De este modo, se forma una cadena de propiedad que se conoce como Blockchain (Huckle, 2016, p. 2). Por lo tanto, el Bitcoin es un tipo de criptomoneda que ha dado lugar a un ámbito de relación social online que permite algo que Marx no pudo suponer: la existencia de un tipo de moneda desvinculada del circuito de producción y circulación del capital.

\subsection{Desintermediación, horizontalismo y confianza}

Igual que la teoría del dinero de Marx está inserta en su teoría del valor-trabajo, es imposible desvincular la creación del Bitcoin de su red de producción y distribución, Blockchain. Igual que el uso del dinero en la teoría de Marx implica una relación concreta e histórica con 
la producción y representación del valor, el Bitcoin y el Blockchain suponen el establecimiento de toda una serie de lógicas de relación social diferentes a aquellas que describe Marx. Por eso, es posible afirmar que el Bitcoin no es tanto una forma de criptomoneda sino un tipo de ideología (Dodd, 2017, pp.1-3).

El conjunto que forman el Bitcoin y el Blockchain puede caracterizarse por tres rasgos principales: la desintermediación, el horizontalismo y la confianza. En primer lugar, el Bitcoin y el Blockchain aparecen bajo una ideología claramente de rechazo de la mediación de tres instituciones en las relaciones sociales y en la vida social: los bancos privados y nacionales y los Estados. La pérdida de confianza en las instituciones financieras, y en la incapacidad regulatoria o de control de las instituciones públicas, hace que el Bitcoin nazca bajo la idea de que es necesario buscar fórmulas de relación social que puedan superar, en la práctica, dicho control (Nakamoto, 2009, pp. 6-8). Más en concreto, se podría decir que el Bitcoin y el Blockchain buscan superar dos formas de mediación (Brunton, 2019; North, 2007; Popper, 2016; Tapscott, 2016): por un lado, la desintermediación del dinero a través de una forma de desnacionalización del mismo, convirtiéndolo en un tipo de relación económica no controlada por el Estado y, por otro lado, y como consecuencia de esto, la superación del control y producción del dinero que tienen los bancos privados a través de la creación de deuda por medio del préstamo de dinero (Dodd, 2017, pp. 4-6).

La segunda característica es el horizontalismo en el que se desarrolla el Bitcoin y el Blockchain. A pesar de que la forma en la que se crean los Bitcoins y la forma en la que se registran sus transacciones en Blockchain es un proceso algo complejo desde el punto de vista técnico (Franco, 2015; Hayes, 2019; Nakamoto, 2009), lo cierto es que existen todo tipo de mecanismos para controlar que no pueda existir ni un acaparamiento de criptomonedas en pocas manos ni que las transacciones puedan ser falseadas. El horizontalismo que se desarrolla en Blockchain permite que todos los miembros de la red, todos los nodos, tengan un acceso igual de directo y transparente al conjunto de todas las transacciones que se desarrollan dentro de la red (Tozzi, 2019).

El tercer elemento es el de la confianza, el cual está directamente relacionado con el esquema de la horizontalidad. Si los bancos centrales y los Estados son los garantes de que el dinero que tenemos en nuestros bolsillos funcione para los mismos propósitos que el dinero real, ¿cómo se garantiza la misma seguridad en la red Blockchain y en la producción de Bitcoins? Nakamoto soluciona este problema a través de lo que denomina un "servidor de marca de tiempo" (timestamp server):

La solución que proponemos comienza con un servidor de marca de tiempo. Este funciona cogiendo un resumen criptográfico de un bloque de ítems a los que se les adjudica una marca de tiempo, publicándose a continuación dicho resumen criptográfico, igual que ocurre en un periódico. La marca de tiempo prueba que los datos deben de haber existido en el tiempo, obviamente, para que se pudieran convertir en un resumen criptográfico. Cada marca de tiempo incluye las marcas de tiempo anteriores en su resumen criptográfico, formando una cadena, en la cual cada marca de tiempo adicional refuerza las anteriores (Nakamoto, 2009, p. 2).

La confianza del Estado y de los bancos nacionales se sustituye por una especie de libro de contabilidad universal en el cual todos los miembros de la red, potencialmente cualquiera que quiera entrar en ella, puede saber en todo momento quien, y qué cantidad, de Bitcoins tiene y la cantidad y el momento de todas sus transacciones. Así, se elimina la necesidad de tener un tercer elemento fuera de la relación social peer-to-peer que sancione, o asegure, la veracidad de una transacción, o creación, de Bitcoins.

Sin embargo, la relación entre el Bitcoin y el Blockchain con la autonomía que tienen con respecto a los bancos públicos y privados, y con respecto al Estado, es ambivalente. Especialmente, en un sentido muy concreto: a pesar de que nació como una forma de producir intercambios comerciales más allá, o por debajo, del control y regulación del sector financiero público-privado, lo cierto es que han acabado por verse insertos en el mismo circuito de especulación del dinero real.

Otro aspecto ambivalente de la autonomía del Bitcoin y del Blockchain descansa en que su carácter autónomo ha hecho que sea un tipo de moneda usada para transacciones comerciales ilícitas o ilegales. Así, dentro de la dark web, el Bitcoin se usa como el dinero virtual perfecto para poder comprar y vender armas, drogas o, incluso, seres humanos. De este modo, la autonomía se convierte en una ventaja para operar al margen del control financiero mundial. Su supuesta capacidad de pasar desapercibida al circuito del dinero se transforma aquí en posibilidad de llevar a cabo operaciones criminales de una forma más efectiva.

Pero existe un tercer elemento que es mucho más importante que el anterior, y que tiene que ver con el proyecto de emancipación a partir del cual se fijen los criterios de una sociedad emancipada o, como en este caso, de una sociedad postcapitalista. La autonomía del Bitcoin (por lo menos en sus comienzos) supone una forma de emancipación con respecto a las formas en las que el Estado controla el intercambio y flujo de dinero. Sin embargo, no supone una superación de la forma capitalista del mercado. Bitcoin puede suponer un elemento de emancipación en la única medida en que entendamos al Estado, y el control que ejerce sobre la economía, como un elemento de coerción de la libertad social de producir e intercambiar riquezas; pero no lo será si entendemos que es el Estado el que garantiza el control de un mercado capitalista dentro de unos ciertos márgenes de racionalidad (Fernández Liria, 2016).

\subsection{Socialismo y libertarianismo}

Por lo tanto, el Bitcoin y el Blockchain nacen bajo la idea de que elementos como la desintermediación, el horizontalismo y la confianza son deseables para una nueva forma de relación social que busque superar la 
doble determinación de los bancos centrales y privados y del Estado. Sin duda, por este motivo se ha visto que dichos elementos caen bajo una perspectiva libertaria, en la cual la construcción de la sociedad emancipada pasa por la creación de espacios de poder autónomos del Estado (Dodd, 2017, pp. 6-7). Esta forma de entender el carácter libertario del Bitcoin y del Blockchain supone también una ambivalencia, ya que es posible entender esa autonomía en un doble sentido.

Por un lado, es posible entenderla desde una perspectiva anarcocapitalista (Golumbia, 2016), desde la cual lo que se busca es que el individuo pueda desarrollar sus negocios, su compraventa de propiedades y sus intercambios de información sin el control del Estado (Casey, 2015; Knapp, 2013; Von Mises, 2009). En esta perspectiva, el individuo, entendido como célula social indisoluble, es autosuficiente para poder desarrollar una vida plena, siendo el Estado simplemente un instrumento de limitación de dicha libertad individual. No obstante, por otro lado, es posible entender que el Blockchain y el Bitcoin permiten la creación de nuevas formas de comunidades y de intercambio de información que, de otro modo, serían imposibles.

Pero la ambivalencia no acaba aquí. Existe un elemento que relaciona al Bitcoin y al Blockchain con la teoría del valor-trabajo de Marx. Desde el punto de vista de la perspectiva más anarcocapitalista, el Bitcoin se entiende bajo la idea de que no existe un valor intrínseco para cada unidad de criptomoneda. Dicho de otro modo: Bitcoin vendría a confirmar una teoría subjetivista del valor en la que, igual que ocurre con el dinero real, el dinero es solo una abstracción (Lotz, 2014a, 2014b, 2015; Neary/ Taylor, 1998; Postone, 2009; Toscano, 2008), un símbolo, un producto humano cuyo valor viene dado por el acuerdo al que dos individuos llegan sobre el valor de un objeto determinado en una circunstancia determinada.

Pero la relación entre valor y Bitcoin tiene otra cara. La inclusión de cierta cantidad de trabajo socialmente necesario es lo que, según Marx, da la posibilidad de poder establecer el valor entre dos mercancías, las cuales, siguiendo la cadena histórico-conceptual, dan lugar al dinero. No obstante, ciertas lecturas socialistas del Bitcoin plantean la posibilidad de que es posible sustituir la cantidad de trabajo socialmente necesario por la energía consumida necesaria para la producción de un Bitcoin (Burns, 2017; Huckel \& White, 2016):

Asumiendo el consumo total de energía de 385.84 $\mathrm{MWh}$, que hemos mencionado antes, y que cada 10 minutos se genera un bloque, un solo bloque usa la siguiente cantidad de energía:

$385.84 \mathrm{MWh} /(1 \mathrm{~h} / 10 \mathrm{~min}) \approx 64.31 \mathrm{MWh}$

Los mineros de Bitcoin reciben una recompensa de 12.5 Bitcoins por generar un bloque. Por lo tanto, la energía que se necesita para un solo Bitcoin es la siguiente:

$64.31 \mathrm{MWh} / 12.5 \approx 5.14 \mathrm{MWh}$ por Bitcoin

(Huckle/White, 2016, p. 10).

De este modo, sería posible calcular el valor de cualquier bien. En una sociedad socialista, en la que fuera necesario, y posible, el cálculo exacto de los precios de los bienes, y en la que, por ejemplo, se quisiera, o se tuviera, que eliminar la dependencia de los combustibles fósiles, se eliminaría el transporte basado en esta forma de energía. De este modo, los vehículos eléctricos se convertirían en algo habitual, así como la producción de electricidad a través de energía renovable. Así, sería posible calcular el precio de un vehículo determinado teniendo en cuenta su producción, transporte, uso y eliminación en relación con el esquema anterior de la cantidad de energía que es necesaria para producir un Bitcoin (Huckle/White, 2016, p. 10).

\subsection{Límites del bitcoin y del blockchain}

A pesar de que el Bitcoin y el Blockchain favorecen la creación de relaciones sociales horizontales y descentralizadas, lo cierto es que también es posible hacerles toda una serie de críticas desde el punto de vista de un proyecto de emancipación social. En primer lugar, es obvio que el Bitcoin y el Blockchain no se han convertido en un sustituto real y generalizado ni del dinero ni de las formas en las que se intercambian derechos de propiedad o se realizan transacciones económicas. A corto plazo parece muy difícil que el Bitcoin sustituya al dinero real (Dodd, 2017, p. 4).

Otro de los inconvenientes más señalados es el de la cantidad de energía que es necesaria para la producción de Bitcoins. La cantidad de energía necesaria para producir un solo Bitcoin es mucho mayor que la necesaria para hacer una transacción con una tarjeta de crédito. La necesidad de una fuerza computacional muy elevada, la cual también limita la producción de criptomoneda a los ordenadores con mayor capacidad de procesamiento, se traduce en un gasto absolutamente desmesurado de energía eléctrica. A pesar de esas lecturas que entienden la cantidad de energía como una forma de sustituir el cálculo del trabajo socialmente necesario en la teoría del valor-trabajo de Marx, lo cierto es que al tomar dicho criterio lo que se hace es ignorar un consumo de energía que es absolutamente incompatible con una conciencia ecológica (Dapprich y Cockshott, 2017).

Un tercer elemento, y tal vez el que se puede aplicar de una forma más general tanto a la forma de funcionamiento del Blockchain como al tipo de relación social que produce, tiene que ver con el supuesto horizontalismo de las relaciones sociales mencionado más arriba. Este podría denominarse el problema político del Blockchain. Un estudio más detallado de las dinámicas online de la producción y distribución del Bitcoin arroja el resultado de que aquellos nodos con mayor capacidad computacional son aquellos que se ven privilegiados en la red. Al necesitarse una capacidad de computación muy importante para producir Bitcoins y para participar en la construcción de bloques, al final aquellos nodos que tengan una menor capacidad de procesamiento se verán excluidos del proceso (Dodd, 2017, pp. 11-13).

Estos serían los elementos de ambivalencia en relación con el aspecto emancipatorio que tienen el 
Bitcoin y el Blockchain. Sin embargo, existe otro conjunto de problemas que tienen que ver con la congruencia entre la teoría del valor-trabajo de Marx y la creación y proceso de circulación del Bitcoin. Dicho de otro modo: ¿qué relación puede establecerse entre el Bitcoin y el Blockchain y el socialismo como proyecto de emancipación?

El punto de relación es el concepto de Marx de créditos de trabajo, es decir, la forma en la que un trabajador vería representado su tiempo de trabajo en una sociedad socialista. La tecnología del Blockchain podría servir para este fin. En una sociedad comunista, los trabajadores recibirían créditos de trabajo por las horas de trabajo realizadas. Más en concreto, cada hora de trabajo sería representada por un cupón que podría ser intercambiado por un producto para el que se haya necesitado la misma cantidad de tiempo de trabajo. De este modo, los Bitcoins podrían servir como cupones digitales de trabajo. Además el Blockchain funcionaría como la herramienta necesaria para llevar a cabo una contabilidad mucho más eficiente de los tiempos de trabajo individuales que la que era posible en el contexto de desarrollo tecnológico en el que escribió Marx (Huckle y White, 2016).

Sin embargo, esta forma de entender el papel del Bitcoin y del Blockchain en un modelo comunista de sociedad tiene un problema fundamental. Los cupones de trabajo, tal y como son conceptualizados por Marx, no necesitan del trabajo para ser producidos. Simplemente, son objetos creados para 'representar' cierta cantidad de tiempo. Este problema consiste en que el Bitcoin no representa una cantidad de tiempo de trabajo determinado, sino la cantidad de energía que él mismo representa. El cupón de trabajo sería la representación de una cierta cantidad de tiempo de trabajo, igual que el dinero real representa una cierta cantidad de tiempo de trabajo. Pero la cantidad de energía que representa el Bitcoin no supone un avance en relación con la superación del verdadero problema que detecta Marx en el dinero: cierta cantidad de tiempo de trabajo 'robado' al trabajador.

\section{Conclusiones}

El Bitcoin y el Blockchain nacieron como una alternativa real a la crisis financiera de 2008 y a la pérdida generalizada de confianza en sus protocolos de actuación. En estos 12 años de desarrollo, sus resultados han sido ambivalentes: por un lado, se ha conseguido crear una moneda virtual que en ciertos ámbitos ha llegado a convertirse en alternativa a las transacciones comerciales con dinero real. También, la red Blockchain ha conseguido establecer ciertas relaciones sociales horizontales y descentralizadas, no solo para la creación de dinero sino para servir de alternativa online a todas aquellas relaciones sociales en las que el Estado o los bancos privados servían como tercera parte sancionadora de un acuerdo concreto.

Por otro lado, el Bitcoin y el Blockchain no han conseguido su propósito principal, que no era otro que servir de alternativa efectiva y real, mainstream, al dinero efectivo. Tampoco han conseguido crear relaciones netamente horizontales dentro de la red, ya que la capacidad computacional sirve como criterio inmanente para permitir o descartar la participación en red. Otros problemas como el uso desmesurado de energía para la producción de Bitcoins suponen serios inconvenientes para que el Bitcoin y el Blockchain supongan, hoy en día, una alternativa efectiva y real al modo de producción capitalista.

Sin embargo, estos inconvenientes no eliminan la necesidad de que se ensayen alternativas a dicha forma hegemónica de producción de riquezas. A pesar de que reproducen toda una serie de problemas políticos, sociales y de organización democrática, lo cierto es que suponen, al menos, un paso en un ensayo hacia nuevas formas de sociedad, el cual parte de dos ventajas muy importantes: que la emancipación social no viene de una vuelta al estatismo y a la cesión de la libertad individual y colectiva, y que la conciencia del proyecto de emancipación de las próximas décadas debe pasar por el uso anticapitalista de la tecnología disponible, idea esta que se encuentra de una forma bastante parecida en el centro mismo de la teoría del valor-trabajo de Marx.

\section{Referencias}

Ammous, S. (2018). The Bitcoin Standard. The Decentralized Alternative to Central Banking. New Jersey: Wiley.

Bjerg, O. (2016). How is Bitcoin money? Theory, Culture \& Society, 33(1), 53-72. https://doi.org/10.1177/2F0263276415619015.

Brunton, F. (2019). Digital Cash. The Unknown History of the Anarchists, Utopians, and Technologists Who Built Cryptocurrency. Princeton/Oxford: Princeton University Press.

Burns, S.A. (2017). Bitcoin and the Marxist Theory of Value. AIER. American Institute for Economic Research. Recuperado de: https://www.aier.org/article/bitcoin-and-the-marxist-theory-of-value/.

Casey, J.M. (2015). The Age of Cryptocurrency: How Bitcoin and Digital Money Are Challenging the Global Economic Order. New York: St. Martins Press.

Dapprich, J. P. y Cockshott, P. Bitcoin is not what socialism needs. Paul Cockshott's Blog. Recuperado de: https://paulcockshott. wordpress.com/2017/11/23/bitcoin-is-not-what-socialism-needs/.

Davidson S., De Filippi, P. y Potts, J. (2018). Blockchains and the economic institutions of capitalism. Journal of Institutional Economics, 14(4), 639-658. https://doi.org/10.1017/S1744137417000200.

De Brunhoff, S. (1973). Marx on Money. New York: Urizen Books.

Di Muzio, T. y Robbins, R. H. (2017). An Anthropology of Money. A Critical Introduction. New York/London: Routledge.

Dodd, N. (2017). The Social Life of Bitcoin. Theory, Culture \& Society, 35(3), 35-56. https://doi.org/10.1177/0263276417746464.

Fernández Liria, C. (2016). En defensa del populismo. Madrid: Los Libros de la Catarata. 
Franco, P. (2015). Understanding Bitcoin. Cryptography, Engineering, and Economics. Cornwall: Wiley.

Golumbia, D. (2016). The Politics of Bitcoin. Software as Right-Wing Extremism. Minneapolis: University of Minnesota Press.

Hayes, A. (2019). The Socio-Technological Lives of Bitcoin. Theory, Culture \& Society, 36(4), 49-72. https://doi. org/10.1177/0263276419826218.

Huckle, S., White, M. (2016). Socialism and the Blockchain. Futures Internet, 8(49), https://doi.org/10.3390/fi8040049

Kennedy, P. (2006). Marx on Commodity-money and the Development of Capital. Critique: Journal of Socialist Theory, 34(1), 27-38. https://doi.org/10.1080/03017600600591681.

Knapp, G. F. (2013). The State Theory of Money. Eastford, CT: Martino Fine Books.

Lange, E. L. (2019). Money versus Value? Reconsidering the 'Monetary Approach' of the 'post'-Uno School, Benetti/Cartelier, and the Neue Marx-Lektüre. Historical Materialism, 28(1), 1-34. https://doi.org/10.1163/1569206X-00001851.

Lotz, C. (2014a). The Transcendental Force of Money: Social Synthesis in Marx. Rethinking Marxism, 26(1), 130-139. https:// doi.org/10.1080/08935696.2014.857851.

Lotz, C. (2015). Marx contra Negri: Value, Abstract Labor, and Money. En Interventions. Contemporary Political Italian Philosophy (A. Calcagno, pp.217-343). New York: SUNY Press.

Lotz, C. (2014b). The Capitalist Schema: Time, Money, and the Culture of Abstraction. Landham, MD: Lexington Books.

Marx, K. (1991). MEGA². Das Kapital. Kritik der Politischen Ökonomie. Erster Band, Hamburg, 1890. Berlin: Dietz Verlag.

Marx, K. (1982). MEGA². Werke, Artikel, Entwürfe. März 1843 bis August 1844. Berlin: Dietz Verlag.

Moseley, F. (ed.). (2005). Marx's Theory of Money. Modern Appraisals. New York: Palgrave McMillan.

Nakamoto, S. (2009). Bitcoin: A Peer-to-Peer Electronic Cash System. Bitcoin.org. Recuperado de: https://bitcoin.org/bitcoin.pdf

Neary, M. y Taylor, G. (1998). Marx and the Magic of Money: Towards an Alchemy of Capital. Historical Materialism, 2(1), 99-117. https://doi.org/10.1163/156920698100414202.

Nelson, A. (1999). Marx's Concept of Money. The God of Commodities. London/New York: Routledge.

North, P. (2007). Money and Liberation. The Micropolitics of Alternative Currency Movements. Minneapolis/London: University of Minnesota Press.

Popper, N. (2016). Digital Gold: Bitcoin and the Inside Story of the Misfits and Millionaires Trying to Reinvent Money. New York: HarperCollins.

Postone, M. (2009). Labor and the Logic of Abstraction: An Interview. South Atlantic Quarterly, 108(2), 305-330. https://doi. org/10.1215/00382876-2008-035.

Saad-Filho, A. (2019). Value and Crisis. Essays on Labour, Money and Contemporary Capitalism. Leiden/Boston: Brill.

Tapscott, D. (2016). Blockchain Revolution: How the Technology Behind Bitcoin Is Changing Money, Business, and the World. New York: Penguin Publishing Grou P.

Toscano, A. (2008). The culture of abstraction. Theory, Culture \& Society, 25(4), 57-75. https://doi.org/10.1177/0263276408091983.

Tozzi, C. (2019). Decentralizing democracy: approaches to consensus within Blockchain communities. Teknokultura. Revista de Cultura Digital y Movimientos Sociales, 16(2), 181-195. http://dx.doi.org/10.5209/TEKN.64523.

Villareal Robledo, O. E.(2016). The Ontological Sociology of Cryptocurrency: A Theoretical Exploration of Bitcoin. (Tesis doctoral, University of Central Florida). Recuperado de: https://stars.library.ucf.edu/cgi/viewcontent.cgi?article=6119\&context=etd.

Von Mises, L. (2009). The Theory of Money and Credit. Alabama: Mises Institut. 